\title{
Correction \\ Correction: da Costa et al. Establishment of a Temperature-Sensitive Model of Oncogene-Induced Senescence in Angiosarcoma Cells. Cancers 2020, 12, 395
}

\author{
Adilson da Costa ${ }^{1,2}$ (), Michael Y. Bonner ${ }^{3}$, Shikha Rao ${ }^{1}$, Linda Gilbert ${ }^{1,4}$, Maiko Sasaki ${ }^{4}{ }^{(0)}$, Justin Elsey ${ }^{1}$, \\ Jamie MacKelfresh ${ }^{1,5}$ and Jack L. Arbiser $1,4,6, *$
}

1 Department of Dermatology, Emory University School of Medicine, Atlanta, GA 30322, USA; adilson_Costa@hotmail.com (A.d.C.); srao30@emory.edu (S.R.); lcgilbert@yahoo.com (L.G.); Justin.elsey@emory.edu (J.E.); jpbower@emory.edu (J.M.)

2 Department of Post-Graduate Studies, Instituto de Assistência Médica ao Servidor Público Estadual, Sao Paulo 04029-000, SP, Brazil

3 Department of Medical Biochemistry \& Biophysics, Karolinska Institutet, 17177 Solna, Sweden; Michael.Bonner@ki.se

4 Atlanta Veterans Affairs Medical Center, Decatur, GA 30033, USA; mpapke@emory.edu

5 Department of Pathology, Emory University School of Medicine, Atlanta, GA 30322, USA

6 Winship Cancer Institute of Emory University, Atlanta, GA 30322, USA

* Correspondence: jarbise@emory.edu; Tel.: +1-(404)-727-5063; Fax: +1-(404)-727-0923

check for

updates

Citation: da Costa, A.; Bonner, M.Y.; Rao, S.; Gilbert, L.; Sasaki, M.; Elsey, J.; MacKelfresh, J.; Arbiser, J.L.

Correction: da Costa et al.

Establishment of a

Temperature-Sensitive Model of

Oncogene-Induced Senescence in

Angiosarcoma Cells. Cancers 2020, 12, 395. Cancers 2021, 13, 2015. https:// doi.org/10.3390/cancers13092015

Received: 15 March 2021

Accepted: 16 March 2021

Published: 22 April 2021

Publisher's Note: MDPI stays neutral with regard to jurisdictional claims in published maps and institutional affiliations.

\section{Copyright: (C) 2021 by the authors.} Licensee MDPI, Basel, Switzerland. This article is an open access article distributed under the terms and conditions of the Creative Commons Attribution (CC BY) license (https:// creativecommons.org/licenses/by/ $4.0 /)$.
The authors wish to make the following corrections to this paper [1]:

The following words in bold face are to be added: In Section 2.1 "Cells", lines 3 and 4: $1 \%$ complex of antibiotics $/ \mathrm{L}$-glutamine (stock $=10,000 \mathrm{IU} / \mathrm{mL}$ penicillin, $10,000 \mu \mathrm{g} / \mathrm{mL}$ streptomycin, and $29.2 \mathrm{mg} / \mathrm{mL}$ L-glutamine; Mediatech Inc., Manassas, VA, USA); in Section 2.4 "Western Blot (WB)", lines 5 and 7: "tri-buffered saline" should be changed to "Tris-buffered saline" on both lines.

In the original article, there was a mistake in Figure 3, where the MW of $\beta$-actin was incorrectly recorded as $65 \mathrm{kd}$, when it should be changed to $45 \mathrm{kd}$. The new Figure 3 is listed below:

\begin{tabular}{lll|l|l} 
& & SVR 37 & $\begin{array}{c}\text { SVR 39 } \\
24 \mathrm{C}\end{array}$ & $\begin{array}{c}\text { SVR 39 } \\
48 \mathrm{hs}\end{array}$ \\
\hline IRF-7 & $65 \mathrm{kDa}$ & & & \\
\hline Stat-1 & $91 \mathrm{kDa}$ & & & \\
\hline IFN- $\alpha$ & $21.5 \mathrm{kDa}$ & & & \\
\hline$\beta$-actin & $45 \mathrm{kDa}$ & & & \\
\hline
\end{tabular}

\begin{tabular}{|c|c|c|c|}
\hline Gene chip microarray & Fold changes & Folds & p-value \\
\hline \multirow{3}{*}{ IRF-7 } & SVR $37^{\circ} \mathrm{C}$ vs SVR $39^{\circ} \mathrm{C} 24 \mathrm{hs}$ & +1.35388 & 0.000425 \\
\hline & SVR $37^{\circ} \mathrm{C}$ vs SVR $39^{\circ} \mathrm{C} 48 \mathrm{hs}$ & +2.30809 & 0.000000245377 \\
\hline & SVR $37^{\circ} \mathrm{C} 24 \mathrm{hs}$ vs SVR $39^{\circ} \mathrm{C} 48 \mathrm{hs}$ & +1.7048 & 0.00000765588 \\
\hline \multirow{3}{*}{ Stat-1 } & SVR $37^{\circ} \mathrm{C}$ vs SVR $39^{\circ} \mathrm{C} 24 \mathrm{hs}$ & +1.75043 & 0.000000249912 \\
\hline & SVR $37^{\circ} \mathrm{C}$ vs SVR $39^{\circ} \mathrm{C} 48 \mathrm{hs}$ & +4.39483 & 0.000000000115134 \\
\hline & SVR $37^{\circ} \mathrm{C} 24 \mathrm{hs}$ vs SVR $39^{\circ} \mathrm{C} 48 \mathrm{hs}$ & +2.51071 & 0.0000501962 \\
\hline \multirow{3}{*}{ IFN- $\alpha$} & SVR $37^{\circ} \mathrm{C}$ Vs SVR $39^{\circ} \mathrm{C} 24 \mathrm{hs}$ & -1.01112 & 0.889057 \\
\hline & SVR $37^{\circ} \mathrm{C}$ vs SVR $39^{\circ} \mathrm{C} 48 \mathrm{hs}$ & +1.0677 & 0.418356 \\
\hline & SVR $37^{\circ} \mathrm{C} 24 \mathrm{~h}$ v vs SVR $39^{\circ} \mathrm{C} 48 \mathrm{hs}$ & +1.07957 & 0.347864 \\
\hline \multirow{3}{*}{$\beta$-actin } & SVR $37^{\circ} \mathrm{C}$ vs SVR $39^{\circ} \mathrm{C} 24 \mathrm{hs}$ & -1.02132 & 0.804895 \\
\hline & SVR3 $37^{\circ} \mathrm{C}$ vs SVR $39^{\circ} \mathrm{C} 48 \mathrm{hs}$ & -1.02549 & 0.768428 \\
\hline & SVR $37^{\circ} \mathrm{C} 24 \mathrm{hs}$ vs SVR $39^{\circ} \mathrm{C} 48 \mathrm{hs}$ & -1.00408 & 0.961911 \\
\hline
\end{tabular}

In Figure 4 , 'B-actin' should be changed to ' $\beta$-actin'. The new Figure 4 is listed below: 


\begin{tabular}{|c|c|c|c|c|c|}
\hline & & SVR $37^{\circ} \mathrm{C}$ & $\begin{array}{c}\text { SVR } 39^{\circ} \mathrm{C} \\
24 \mathrm{hs}\end{array}$ & $\begin{array}{c}\text { SVR } 39^{\circ} \mathrm{C} \\
48 \mathrm{hs}\end{array}$ & \\
\hline $80 \mathrm{kDa}$ & \multicolumn{2}{|l|}{ cNotch1 } & & & \\
\hline 300kDa & \multicolumn{2}{|c|}{ Notch1 (full length) } & & & \\
\hline 120kDa & \multicolumn{2}{|c|}{ Notch1 (transmembrane) } & & & \\
\hline 22kDa & \multicolumn{2}{|c|}{ Presenilin 1} & (a) & & \\
\hline $23 \mathrm{kDa}$ & \multicolumn{2}{|c|}{ Presenilin 2} & 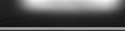 & & \\
\hline $45 \mathrm{kDa}$ & \multicolumn{2}{|l|}{$\beta$-actin } & & & \\
\hline \multicolumn{2}{|l|}{ Gene chip microarray } & Fold changes & \multicolumn{2}{|c|}{ Folds } & p-value \\
\hline \multirow{3}{*}{\multicolumn{2}{|c|}{ Notch1 }} & SVR $37^{\circ} \mathrm{C}$ vs SVR $39^{\circ} \mathrm{C} 24 \mathrm{hs}$ & \multicolumn{2}{|l|}{-1.01248} & 0.799102 \\
\hline & & SVR $37^{\circ} \mathrm{C}$ vs SVR $39^{\circ} \mathrm{C} 48 \mathrm{hs}$ & \multicolumn{2}{|l|}{+1.27551} & 0.000859 \\
\hline & & SVR $37^{\circ} \mathrm{C} 24 \mathrm{hs}$ vs SVR $39^{\circ} \mathrm{C} 48 \mathrm{hs}$ & \multicolumn{2}{|l|}{+1.29142} & 0.000625 \\
\hline \multirow{3}{*}{ Presenilin 1} & & SVR $37^{\circ} \mathrm{C}$ vs SVR $39^{\circ} \mathrm{C} 24 \mathrm{hs}$ & \multicolumn{2}{|l|}{-1.01063} & 0.73016 \\
\hline & & SVR $37^{\circ} \mathrm{C}$ vs SVR $39^{\circ} \mathrm{C} 48 \mathrm{hs}$ & \multicolumn{2}{|l|}{-1.32619} & 0.000012112 \\
\hline & & SVR $37^{\circ} \mathrm{C} 24 \mathrm{hs}$ vs SVR $39^{\circ} \mathrm{C} 48 \mathrm{hs}$ & \multicolumn{2}{|l|}{-1.31223} & 0.0000160651 \\
\hline \multirow{3}{*}{\multicolumn{2}{|c|}{ Presenilin 2}} & SVR $37^{\circ} \mathrm{C}$ vs SVR $39^{\circ} \mathrm{C} 24 \mathrm{hs}$ & \multicolumn{2}{|l|}{-1.13006} & 0.087376 \\
\hline & & SVR $37^{\circ} \mathrm{C}$ vs SVR $39^{\circ} \mathrm{C} 48 \mathrm{hs}$ & \multicolumn{2}{|l|}{-1.0685} & 0.322156 \\
\hline & & SVR $37^{\circ} \mathrm{C} 24 \mathrm{hs}$ vs SVR $39^{\circ} \mathrm{C} 48 \mathrm{hs}$ & \multicolumn{2}{|l|}{+1.05761} & 0.398453 \\
\hline \multirow{3}{*}{$\beta$-actin } & & SVR $37^{\circ} \mathrm{C}$ vs SVR $39^{\circ} \mathrm{C} 24 \mathrm{hs}$ & \multicolumn{2}{|l|}{-1.02132} & 0.804895 \\
\hline & & SVR $37^{\circ} \mathrm{C}$ vs SVR $39^{\circ} \mathrm{C} 48 \mathrm{hs}$ & \multicolumn{2}{|l|}{-1.02549} & 0.768428 \\
\hline & & SVR $37^{\circ} \mathrm{C} 24 \mathrm{hs}$ vs SVR $39^{\circ} \mathrm{C} 48 \mathrm{hs}$ & -1.00408 & & 0.961911 \\
\hline
\end{tabular}

In Figure 5, the SDHA band was accidently duplicated and listed as p53. The duplicated band has been removed. The new Figure 5 is listed below:

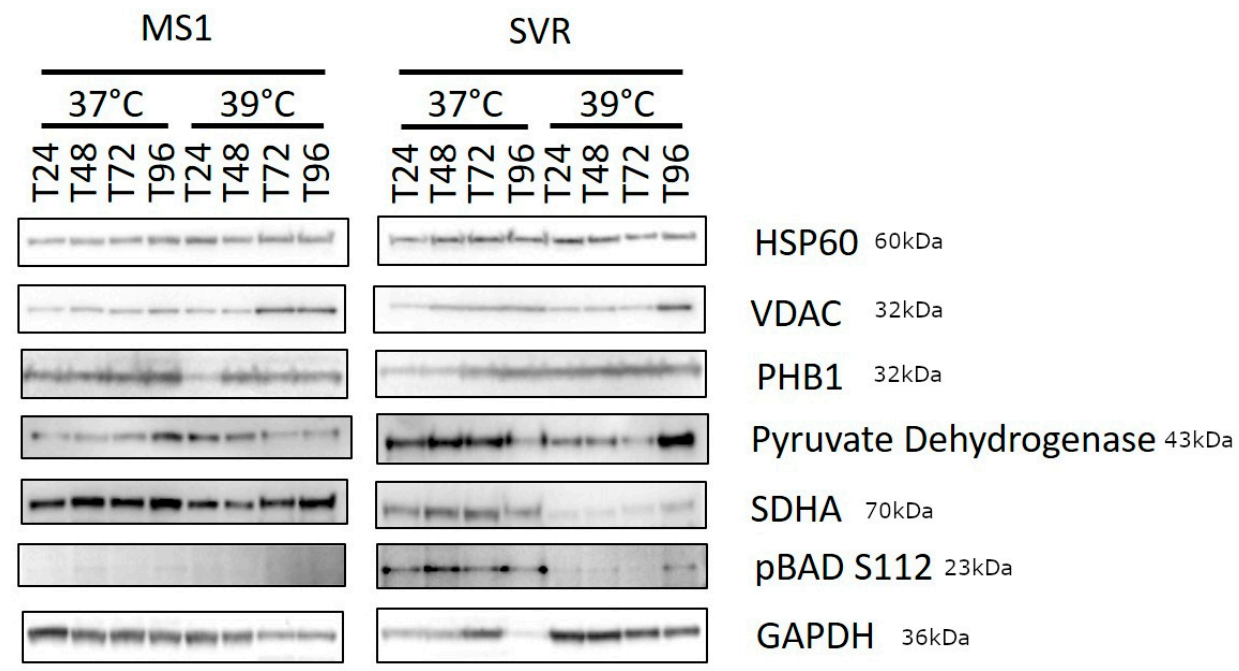

The p53 figure represents a duplication of the SDHA Western blot, and thus should be deleted. Since we are not using the p53 data, we request the deletion of the following sentences: "p53 is dephosphorylated in SVR cells upon shifting to the nonpermissive temperature in ras transformed SVR cells, but not in MS1 cells, suggesting that this is not a nonspecific heat shock event, but that it is induced by oncogenic ras" in the last 
paragraph of Section 3 "Result" and the sentence "p53 is rapidly phosphorylated in SVR cells upon shift to $39^{\circ} \mathrm{C}$ (Figure 5) and it is well known that phosphorylated p53 translocates to the mitochondria and may mediate apoptosis [46]" in Section 4 "Discussion and Conclusions" should be removed. Reference [47] and [48] should thus be re-numbered to [46] and [47], respectively.

The authors apologize for any inconvenience caused and state that the scientific conclusions are unaffected. The original article has been updated.

\section{Reference}

1. Da Costa, A.; Bonner, M.Y.; Rao, S.; Gilbert, L.; Sasaki, M.; Elsey, J.; MacKelfresh, J.; Arbiser, J.L. Establishment of a TemperatureSensitive Model of Oncogene-Induced Senescence in Angiosarcoma Cells. Cancers 2020, 12, 395. [CrossRef] [PubMed] 